\title{
SPECIAL ISSUE EDITORIAL ON ADVANCES IN COLLABORATIVE SYSTEMS ENGINEERING FOR PRODUCT DESIGN, PRODUCTION AND SERVICE NETWORK
}

\author{
Amy J.C. Trappey ${ }^{1}$ Weiming Shen ${ }^{2}$ John Jianzhong Cha ${ }^{3}$ \\ ${ }^{l}$ Department of Industrial Engineering and Engineering Management, National Tsing Hua University, \\ Taiwan, China \\ trappey@ie.nthu.edu.tw $(\bowtie)$ \\ ${ }^{2}$ Integrated Manufacturing Technologies Institute, National Research Council Canada, Canada \\ wshen@ieee.org \\ ${ }^{3}$ Department of Mechanical Engineering, Beijing Jiaotong University, China \\ jzcha@bjtu.edu.cn
}

From the perspectives of concurrent engineering (CE), computer supported cooperative work (CSCW), product lifecycle management (PLM), to collaborative supply chain and service network, collaborative systems engineering (CSE) has been extended and enriched in many research efforts. CSE is not just a technique or method but also a fundamental business philosophy and strategy. It provides a consistent set of solutions to support the collaborative co-creation, management, dissemination, and use of information and knowledge through the entire lifecycle of product design, production, and services, which engage and integrate processes, systems, organizations, people, and information effectively and intelligently. During the process of reviewing the submitted papers for this special issue, we have carefully selected six papers providing novel methodologies to enable CSE covering two main categories of collaborative systems, i.e., for product design collaborations and for production and service network collaborations. The first category has two papers focusing on improving collaborative design efficiency using annotated computer aided design (CAD) modelling and bill-of-material (BOM)-based design knowledge representation. The second category includes four papers. They focus on solving challenging issues for enabling the production and service collaborations and their systems, e.g., multi-node tracking for wireless sensor network, agent-based neural network system for failure prediction, one-stop logistic services for collaborative supply chain, and system maintainability analysis for complex collaboration. The scopes and insights of these research papers shall provide valuable references for future research and development of collaborative systems engineering. 


\section{For Product Design Collaboration:}

1. Synchronous communication in PLM environments using annotated CAD models (by Jorge D. Camba, Manuel Contero, Gustavo Salvador-Herranz, Raquel Plumed).

2. BOM-based design knowledge representation and reasoning for collaborative product development (by Gong-Zhuang Peng, Hua-Chao Mao, Hong-Wei Wang, He-Ming Zhang).

\section{For Production and Service Network Collaboration:}

3. Comparing two clustering-based techniques to track mobile nodes in industrial wireless sensor networks (by Mohammad Gholami and Robert W. Brennan).

4. A quantum multi-agent based neural network model for failure prediction (by Wei Wu, Min Liu, Qing Liu, Weiming Shen).

5. A one-stop logistic services framework supporting global supply chain collaboration (by A.J.C. Trappey, C.V. Trappey, S.W.C. Chang, W.T. Lee, T. N. $\mathrm{Hsu})$.

6. Complexity of system maintainability analysis based on the interpretive structural modelling methodology: Transdisciplinary approach (by A. Ertas, M. W. Smith, D. Tate, W. D. Lawson, T. B. Baturalp).

Amy J.C. Trappey is a distinguished university professor of industrial engineering and engineering management at the National Tsing Hua University, Taiwan, China. Dr. Trappey's research interests are in e-business, knowledge engineering and management, particularly focusing on intellectual property and patent analyses. She is an ASME and ISEAM Fellow.

Weiming Shen received his Ph.D. degree from the University of Technology of Compiègne, France, in 1996. He was a Lecturer at the Beijing Jiaotong University from 1986 to 1992. $\mathrm{He}$ is currently a Senior Research Scientist with the National Research Council Canada, Ottawa, Ontario, Canada. He is Adjunct Professor at the University of Western Ontario, London, ON, Canada and Adjunct Professor at the Tongji University, Shanghai, China. $\mathrm{He}$ has been working on intelligent software agents, IoT, and Big Data, and their applications to different engineering domains for many years. He has published several books and over 400 papers in scientific journals and international conferences in the related areas, with over 8000 citations. Dr. Shen is a Fellow of IEEE, a Fellow of the Engineering Institute of Canada, and a Registered Professional Engineer in the Province of Ontario, Canada.

John Jianzhong Cha is a professor of Beijing Jiaotong University (BJTU), the Chair holder and Chairman of the Board of Directors, UNESCO Chair on Cooperation between Higher Engineering Education and Industries at BJTU (since 2005). He serves as a Member of Governing Board of UNESCO Institute for IT in Education (since 2009), a Member of Board of Directors of Group T International Engineering College of University of Leuven of Belgium 
Trappey et al.: Special Issue Editorial on Advances in Collaborative Systems Engineering for Product Design, Production and Service Network

(since 2003). He received his B.S. from Tsinghua University, M.S. from Tianjin University and Ph.D. from SUNY/Buffalo of USA. He worked in industry for more than 10 years. He published over 400 academic papers and 6 books, advised over 80 graduate students at Master, Doctoral and postdoctoral level in the field of intelligent design, control and manufacturing. 